\title{
Morbilidad y mortalidad operatoria de la gastrectomía subtotal y total por cáncer gástrico 2004 a 2010. Parte I de un estudio prospectivo*
}

\author{
Drs. ATTILA CSENDES J. ${ }^{1}$, ITALO BRAGHETTO M. ${ }^{1}$, JUAN CARLOS DÍAZ J. ${ }^{1}$, \\ JAIME CASTILLO K. ${ }^{1}$, JORGE ROJAS C. ${ }^{1}$, E.U. SOLANGE CORTÉS L. ${ }^{1}$ \\ 1 Departamento de Cirugía, Hospital Clínico Universidad de Chile. \\ Santiago, Chile.
}

\begin{abstract}
Complications and mortality of total and subtotal gastrectomy for gastric cancer

Background: Surgery for gastric cancer may have high rates of complications and mortality. Aim: To analyze operative mortality of total and subtotal gastrectomy in the period 2004-2010. Material and Methods: Prospective study 345 patients with gastric cancer, mean age 62 years, 64\% males, subjected to a total or subtotal R0, R1 or R2 gastrectomy. All patients were assessed in the postoperative period and all complications were recorded. Results: Total and subtotal gastrectomies were performed in 224 and 69 patients respectively. Postoperative complications consisted in anastomotic leaks, duodenal stump leaks, hemoperitoneum, pulmonary infections and intestinal obstruction. Mortality of total gastrectomy R1 or R2 was 2.1\% whereas palliative gastrectomy, to improve quality of life, had 15\% mortality. Subtotal gastrectomy had $1.4 \%$ mortality. Conclusions: There has been a reduction in operative mortality of gastrectomy for gastric cancer, however the rate of complications has not changed.
\end{abstract}

Key words: Gastric cancer, mortality, gastrectomy.

\section{Resumen}

Objetivos: Analizar la morbilidad y mortalidad operatoria de la gastrectomía total y subtotal en el período 2004-2010 y compararlo con las cifras publicadas en los últimos 40 años. Material y Método: El presente estudio prospectivo incluye a los pacientes sometidos a gastrectomía total o subtotal $R_{0} R_{1}$ o $R_{2}$ entre mayo de 2004 y diciembre de 2010. Se evaluaron la morbilidad y la mortalidad postoperatoria. Resultados: La gastrectomía total se realizó en 244 pacientes y la subtotal en 69 pacientes. La morbilidad postoperatoria correspondió a filtración de la anastomosis, filtración del muñón duodenal, hemoperitoneo, infecciones pulmonares y obstrucción intestinal. La mortalidad de la gastrectomía total $\mathrm{R}_{0}$ o $\mathrm{R}_{1}$ fue de 2,1\%, mientras que la gastrectomía de aseo tuvo un 15\% de mortalidad. La gastrectomía subtotal presentó una mortalidad de 1,4\%. Conclusiones: Como consecuencia de todos los adelantos técnicos y materiales en la cirugía del cáncer gástrico, que significó una baja muy importante en la mortalidad operatoria, las complicaciones postoperatorias no han variado estos últimos 40 años.

Palabras clave: Cáncer gástrico, gastrectomía, morbilidad, mortalidad.

*Recibido el 4 de abril de 2011 y aceptado para publicación el 6 de junio de 2011.

Correspondencia: Dr. Attila Csendes J.

Santos Dumont 999. Santiago, Chile.

acsendes@redclinicauchile.cl 


\section{Introducción}

El cáncer gástrico aún representa la primera causa de mortalidad por tumores malignos en Chile ${ }^{1}$, por lo tanto, es un muy importante problema de salud pública en todo el país. Exceptuando casos seleccionados de cáncer mucoso menores de $2 \mathrm{~cm}$, en la inmensa mayoría de los pacientes, el único tratamiento efectivo que logra la curación de la enfermedad es la resección oncológica del tumor, que incluye la remoción parcial o total del estómago y una linfadenectomía $\mathrm{D}_{2}^{2,3}$. A pesar de varios trabajos que se han referido a la mortalidad operatoria y la sobrevida de estos pacientes, no hay referencias recientes respecto a las complicaciones médicas y quirúrgicas precoces después de la cirugía, especialmente si se utilizaron técnicas recientes como la completa evaluación nutricional preoperativa, el estadiaje con tomografía axial computada, el uso de suturas mecánicas en forma masiva, alimentación enteral precoz postoperatoria, etc, técnicas que no estaban disponibles en forma rutinaria antes de la década de los 90 . Por lo tanto, el objetivo del presente estudio prospectivo fue determinar las complicaciones médicas y quirúrgicas en pacientes con cáncer gástrico sometidos a una resección quirúrgica, mediante un protocolo de estudio prospectivo y compararlos con los resultados obtenidos por los autores desde 1969 hasta 2004.

\section{Material y Método}

\section{Pacientes estudiados}

En mayo de 2004 se constituyó en el Departamento de Cirugía una Unidad de Oncología a cargo de una enfermera universitaria (S.C.) especializada en cirugía, con el objeto de registrar a todos los pacientes desde su ingreso al departamento, su tratamiento y evolución postoperatoria, las características anátomo patológicas completas y muy en especial el seguimiento de cada paciente cada 6 meses, independiente de los controles médicos. El estudio prospectivo incluye a 345 pacientes con cáncer gástrico sometidos a una intervención quirúrgica, que corresponden a 4,4 pacientes operados por mes y 52 anuales en promedio. De ellos 221 pacientes $(64,1 \%)$ eran hombres y 124 pacientes (35,9\%) eran mujeres, en una proporción de 1,8:1. La edad promedio fue de 62,1 años, siendo menores de 40 años un 6,9\% y sobre 70 años un $32,5 \%$. Se excluyeron pacientes no operados y pacientes con linfoma gástrico (8 casos).

\section{Estudio preoperatorio}

Todos los pacientes tenían un completo estudio preoperatorio que incluía anamnesis y examen físico, exámenes de laboratorio rutinarios completos, panendoscopia y biopsia que demostraba la presencia de un adenocarcinoma, tomografía axial computarizada de abdomen, pelvis. El 33\% de los casos tenían estudio radiológico de esófago, estómago y duodeno. Pacientes con algún grado de déficit nutricional (3\%) fueron sometidos a tratamiento nutricional oral y enteral, excepcionalmente a nutrición parenteral. En ninguno se empleó neoadyuvancia.

\section{Técnica quirúrgica}

La gastrectomía subtotal correspondió a una resección gástrica entre 70 y 80\%, con omentectomía $\mathrm{y}$ anastomosis gastro yeyunal con asa en Y-de-Roux de 60 a $70 \mathrm{~cm}$. Esta anastomosis fue básicamente de tipo manual y se empleó sutura mecánica en un 10\%. La gastrectomía total consistió en la completa remoción del estómago, incluyendo un segmento de esófago abdominal y $2 \mathrm{~cm}$ de duodeno, junto con omentectomía. En estos pacientes se envió de rutina un trozo del límite oral a nivel esofágico a anatomía patológica, para descartar infiltración tumoral del borde oral. La reconstrucción esofagoyeyunal fue con un asa en Y-de-Roux de $70 \mathrm{~cm}$, empleando sutura mecánica circular con Stapler 25 en un 95\% de los $\operatorname{casos}^{3-5}$.

En todos los pacientes sometidos a cirugía se realizó una linfadenectomía D2, resecando un promedio de 32,4 linfonodos por paciente. En los pacientes sometidos a cirugía R1 o R2, la disección linfonodal fue de D1, resecando sólo los linfonodos perigástricos. Se colocaron 2 drenajes peri anastomosis y una sonda nasoyeyunal de rutina, para comenzar la alimentación enteral a las 48 horas de la cirugía.

\section{Evaluación postoperatoria}

Cada paciente fue evaluado constantemente 2 a 3 veces por día por el equipo médico tratante, consignando todas las eventuales complicaciones que aparecían, las que fueron incluidas en una tabla Excel especialmente confeccionada por la enfermera, contando por lo tanto, con toda la morbilidad postoperatoria hasta el alta del paciente.

\section{Etapificación de los pacientes}

Para incluir a cada paciente en un estadio evolutivo, se empleó la clasificación TNM del año 2002.

\section{Cálculo estadístico}

Para la significación estadística se empleó el Test de Fisher, tomando un $\mathrm{p}<0,05$ como significativo.

\section{Mortalidad operatoria}

Se la consideró cuando el fallecimiento ocurrió antes de los 30 días del postoperatorio o durante una hospitalización prolongada hasta más de 30 días. 


\section{Resultados}

La Tabla 1 muestra la distribución, según el estadio, de los 345 pacientes con cáncer gástrico. Se aprecia que en las etapas más precoces (0, IA y IB) hay un $25,8 \%$ de los pacientes, mientras que en etapa avanzada (IV) hay un $37,4 \%$. La Tabla 2 describe los tipos de cirugía realizada. Se aprecia que 2/3 de los pacientes fueron sometidos a gastrectomía total, siendo la resecabilidad total de un $90,8 \%$. La gastrectomía total R2 “de aseo", es decir, la extirpación de todo el estómago, para mejorar la calidad de vida, evitando hemorragias u obstrucción gástrica, se realizó en 13 pacientes (Tabla 3). En estos, se observó una elevada morbilidad y fallecen 2 de los casos, uno por filtración del muñón duodenal y otro por un shock séptico de origen abdominal. Hubo una diferencia muy significativa en los días de hospitalización entre los pacientes complicados o no $(\mathrm{p}<0,001)$. La Tabla 4 señala los resultados postoperatorios en 231 pacientes sometidos a gastrectomía total R0 (214) o R1 (17 pacientes). Hubo un total de $42,9 \%$ de morbilidad postoperatoria. Las complicaciones quirúrgicas más importantes fueron filtración de la anastomosis en 10,4\%, siendo de tipo I 15 pacientes $(6,5 \%)$ y de

Tabla 1. Etapificación de pacientes con cáncer gástrico. $\mathrm{N}=345$

\begin{tabular}{|ccc|}
\hline Estadio & n & \% \\
\hline 0 & 4 & 1,2 \\
\hline IA & 58 & 16,8 \\
\hline IB & 27 & 7,8 \\
\hline II & 41 & 11,9 \\
\hline IIIA & 49 & 14,2 \\
\hline IIIB & 37 & 10,7 \\
\hline IV & 129 & 37,4 \\
\hline
\end{tabular}

Tabla 2. Tipos de cirugía en cáncer gástrico. $\mathrm{N}=345$

\begin{tabular}{|lrl|}
\hline Gastrectomía total & 231 & $(67 \%)$ \\
\hline Gastrectomía subtotal & 69 & $(20 \%)$ \\
Gastrectomía total de aseo & 13 & $(3,8 \%)$ \\
\hline Otras no resectivas & 32 & $(9,2 \%)$ \\
\hline
\end{tabular}

tipo II el 3,9\% (6). Ninguno de estos pacientes se reoperó. Hubo un total de 18 pacientes (7,8\%) que requirieron una reoperación, de los cuales 4 fueron por complicaciones pulmonares (derrame o empiema), 3 por filtración del muñón duodenal, 5 por obstrucción intestinal precoz $(2,2 \%)$ y 4 por hemoperitoneo (1,7\%). La mortalidad operatoria fue de un 2,1\%, correspondiendo a 5 pacientes: Dos por filtración de la anastomosis esófago-yeyunal tipo II, 2 por neumonía nosocomial sin complicación abdominal y 1 paciente por insuficiencia hepática con una cirrosis hepática grado A de Child. Por lo tanto, de los 15 pacientes con filtración tipo I ninguno falleció y de los 9 pacientes con filtración tipo II, fallecen 2 (22\%). Ningún paciente con filtración del muñón duodenal falleció.

La Tabla 5 muestra la morbilidad de la gastrectomía subtotal R0 (56 casos) o R1 (13 casos). Hubo 11 compli-

Tabla 3. Gastrectomía total “de aseo" $\left(R_{2}\right) \cdot N=13$

\begin{tabular}{|lll|}
\hline 1. Complicaciones & $\mathrm{n}=7$ & (53,3\%) \\
Pulmonares & 4 & \\
$\begin{array}{l}\text { Filtración muñón duodenal } \\
\text { Filtración anastomosis }\end{array}$ & 1 (reoperado) & \\
Shock séptico & 1 & \\
2. Mortalidad & 1 & $(15,4 \%)$ \\
$\begin{array}{l}\text { Filtración duodenal } \\
\text { Shock séptico }\end{array}$ & $\mathrm{n}=2$ & \\
$\begin{array}{l}\text { 3. Hospitalización promedio } \\
\text { Sin complicación }\end{array}$ & & \\
Con complicación & $8,8 \pm 1,8$ días & \\
& $32 \pm 29$ días & $\mathrm{p}<0,001$ \\
\hline
\end{tabular}

Tabla 4. Gastrectomía total $R_{0} 0 R_{1} \cdot N=231$

\begin{tabular}{|lll|}
\hline 1. Complicaciones quirúrgicas & $\mathrm{n}=64$ & $(27,8 \%)$ \\
Filtración anastomosis & 24 & $(10,4 \%)$ \\
Colección abdominal & 17 (2 reop) & \\
Filtración muñón duodenal & 8 (3 reop) & \\
Infección herida op & 6 & \\
Obstrucción intestinal & 5 (5 reop) & \\
Hemoperitoneo & 4 (4 reop) & \\
2. Complicaciones médicas & $\mathrm{n}=35$ & $(15,1 \%)$ \\
Pulmonares & 15 (4 reop) & \\
Derrame pleural & 5 & \\
TEP & 1 & \\
Cardiovasculares & 9 & \\
Otros & 5 & $(7,8 \%)$ \\
3. Reoperados & $\mathrm{n}=18$ & $(2,1 \%)$ \\
Mortalidad operatoria & $\mathrm{n}=5$ & \\
5. Días hospitalización & & \\
Sin complicaciones & $9 \pm 1,5$ días & \\
Con complicaciones & $19,7 \pm 19,2$ días & $\mathrm{p}<0,001$ \\
\hline
\end{tabular}

TEP $=$ Tromboembolismo pulmonar. 
Tabla 5. Gastrectomía subtotal $R_{0} \circ R_{1} \cdot N=69$

\begin{tabular}{|c|c|c|}
\hline $\begin{array}{l}\text { 1. Complicaciones quirúrgicas } \\
\text { Colección abdominal } \\
\text { Filtración anastomosis } \\
\text { Filtración muñón duodenal } \\
\text { Estenosis anastomosis } \\
\text { Hemoperitoneo } \\
\text { Infección herida op } \\
\text { Íleo postop }\end{array}$ & $\begin{array}{l}\mathrm{n}=11 \\
3 \\
1 \\
2(1 \text { reop) } \\
2 \\
1 \\
1 \\
1\end{array}$ & \\
\hline $\begin{array}{l}\text { 2. Complicaciones médicas } \\
\text { Delirio postop } \\
\text { Cardiovasculares } \\
\text { Pulmonares }\end{array}$ & $\begin{array}{l}\mathrm{n}=6 \\
2 \\
3 \\
1\end{array}$ & \\
\hline 3. Reoperados & $\mathrm{n}=1$ & $(1,4 \%)$ \\
\hline 4. Mortalidad operatoria & $\mathrm{n}=1$ & $(1,4 \%)$ \\
\hline $\begin{array}{l}\text { 5. Días hospitalización } \\
\text { Sin complicaciones } \\
\text { Con complicaciones }\end{array}$ & $\begin{array}{l}6,6 \pm 1,2 \text { días } \\
14 \pm 0,8 \text { días }\end{array}$ & $\mathrm{p}<0,01$ \\
\hline
\end{tabular}

Tabla 6. Evolución de la mortalidad operatoria en nuestra experiencia

\begin{tabular}{|lccc|}
\hline Autor & Año & Mortalidad GST & Mortalidad GT \\
\hline Otaíza $^{7}$ & 1969 & $19 \%$ & $50 \%$ \\
\hline Csendes $^{8}$ & 1972 & --- & $33 \%$ \\
Csendes $^{9}$ & 1974 & --- & $33 \%$ \\
Csendes $^{10,11}$ & 1975 & $10 \%$ & $27 \%$ \\
Csendes $^{12}$ & 1983 & $15,3 \%$ & $23 \%$ \\
Csendes $^{3}$ & 2002 & --- & $3 \%$ \\
\hline Csendes $^{2}$ & 2011 & $1,4 \%$ & $2,1 \%$ \\
\hline
\end{tabular}

GST = Gastrectomía subtotal. GT = Gastrectomía total.

Tabla 7. Mortalidad operatoria de la gastrectomía total en publicaciones chilenas

\begin{tabular}{|lccc|}
\hline Autor & Año & \multicolumn{1}{c}{ n casos } & $\begin{array}{c}\text { Mortalidad } \\
\text { operatoria (\%) }\end{array}$ \\
\hline Braghetto $^{14}$ & 1989 & 32 & $3(9,3)$ \\
Anselmi $^{15}$ & 1989 & 28 & $5(17,8)$ \\
\hline Álvarez $^{16}$ & 1995 & 104 & $6(5,7)$ \\
Venturelli $^{17}$ & 1996 & 60 & $1(2)$ \\
\hline Burmeister $^{18}$ & 1997 & 546 & $9(1,6)$ \\
\hline Cenitagoya $^{19}$ & 1997 & 134 & $13(9,5)$ \\
\hline Wanghe $^{20}$ & 1999 & 61 & $6(9,8)$ \\
\hline Hernández $^{21}$ & 1999 & 102 & $11(10,7)$ \\
\hline Álvarez $^{22}$ & 1999 & 101 & $7(6,9)$ \\
Álvarez $^{23}$ & 2004 & $163<65$ años & $4(2,5)$ \\
& & $101>65$ años & $7(6,9)$ \\
Valenzuela $^{24}$ & 2004 & 102 & $5(4,9)$ \\
\hline Csendes $^{23}$ & 2011 & 231 & $5(2,1)$ \\
\hline
\end{tabular}

caciones quirúrgicas (16\%) y 6 médicas (8,7\%). Destacan los 3 casos con colecciones abdominales tratadas con drenaje percutáneo por radiología intervencional, 1 paciente con filtración de la anastomosis gastroyeyunal (9\%) de tipo I y 2 filtraciones del muñón duodenal (18\%) uno de ellos, el único reoperado $(1,4 \%)$. La mortalidad operatoria correspondió a este mismo paciente reoperado, quien falleció a los 17 días de la cirugía. El promedio de días de estada hospitalaria fue significativamente menor en pacientes sin complicaciones comparado a los pacientes con morbilidad.

\section{Discusión}

Los resultados del presente estudio sugieren varios hechos: en primer lugar el establecimiento de una unidad de oncología que permite realizar estudios prospectivos muy cuidadosos, es muy superior a las revisiones retrospectivas. En segundo lugar, la notable mejoría en la mortalidad operatoria en nuestro grupo de trabajo, evaluando 40 años de evolución; en tercer lugar, a pesar de todos los adelantos, las principales causas de morbilidad postoperatoria no han cambiado en las últimas 4 décadas.

El presente estudio no se ha referido a la resecabilidad del tumor, que claramente ha aumentado en forma muy significativa, como se ha publicado anteriormente ${ }^{7}$. Este aumento de la resecabilidad es uno de los importantes progresos en el tratamiento del cáncer gástrico. El establecimiento de esta unidad de oncología con registro de todos los pacientes con tumores malignos, dirigido por una enfermera quirúrgica especializada, ha permitido crear una plantilla Excel para cada tipo de tumor, incluyendo todos los parámetros que son importantes para el grupo quirúrgico. La realización de un protocolo prospectivo es inmensamente superior a las revisiones retrospectivas, que adolecen de varios defectos, como la no inclusión de algunos parámetros importantes, el criterio diferente no estandarizado de los cirujanos tratantes, la pérdida de fichas clínicas, seguimiento sólo hasta el "retiro de los puntos de la herida operatoria" sin seguimiento posterior, el desconocimiento si se efectuó una completa evaluación nutricional, etc. En el presente estudio, el establecimiento de este estudio clínico prospectivo ha permitido te- 
ner un seguimiento de $100 \%$ de los pacientes, como se publicará con posterioridad.

La notable disminución de la mortalidad operatoria de nuestro grupo de trabajo se muestra en la Tabla 6, con la evolución en los últimos 40 años de esta en gastrectomía subtotal y total ${ }^{7-13}$. En el primer trabajo $^{8}$, se describe una mortalidad de $50 \%$ para la gastrectomía total y de $19 \%$ para la subtotal. En estudios posteriores esta mortalidad fue bajando lentamente, pero con cifras altas hasta $1983^{13}$. ¿Porqué bajó tanto esta cifra en las últimas décadas? Creemos que esto se debe a 2 hechos principales: "factor primario y factor secundario". En el factor secundario se incluyen los importantes avances en el diagnóstico más precoz, tratamiento nutricional adecuado, manejo kinésico del postoperatorio, medicamentos muy eficaces para controlar infecciones, unidades de cuidado intensivo con ventilación mecánica muy eficiente, etc. El "factor primario" se refiere al cirujano y al grupo quirúrgico. En 1983 se organizó en el departamento de cirugía, siguiendo la tendencia mundial, diversas unidades especializadas en el manejo de las diferentes patologías quirúrgicas. El establecimiento de la unidad de digestivo alto ha resultado en que un grupo de cirujanos se dedicara al tratamiento de enfermedades esófago gástricas, lo que permitió desarrollar el concepto de "alto volumen”, con un débito anual de pacientes que les permitía adquirir experiencia. Esto resulta en una mejor atención del paciente, con experiencia quirúrgica para decidir conducta, conocimiento, diagnóstico y manejo oportuno de las complicaciones que aparecen en el postoperatorio, establecimiento de protocolos de estudio y seguimiento de pacientes, etc. En la Tabla 7 se detalla la mortalidad operatoria de la gastrectomía total de numerosos cirujanos chilenos de diferentes regiones que se han especializado en el manejo de pacientes con cáncer gástrico. En general en todos los grupos se aprecia un importante descenso de la mortalidad, comparando cifras de 1989 hasta $2004^{13-24}$.

Sin embargo, llama la atención que a pesar de todos los avances y adelantos, la morbilidad postoperatoria es la misma, aunque el riesgo de mortalidad ha bajado notablemente. Nuestros estudios de $1975^{11,12}$, mostraban que las principales causas de complicación postoperatoria eran filtraciones de la anastomosis, hemoperitoneo, obstrucción intestinal, infección herida operatoria, filtración de muñón duodenal; junto con importantes complicaciones pulmonares, que son las mismas complicaciones post operatorias en la actualidad. La diferencia está en la mortalidad de estas complicaciones. Por ejemplo, en 1975 hubo un 10\% de filtraciones que significó el fallecimiento de todos ellos y fue la responsable de la mitad de todos los fallecidos ${ }^{6}$. Es muy probable que todas esas filtraciones eran de tipo II, es decir, con manifestaciones clínicas. En el presente estudio las filtraciones tipo II correspondieron a un 3,9\%, falleciendo sólo 2 de los pacientes por cuadro séptico. El resto se manejó médicamente. Clavien et al, en $1992^{25}$, propuso una clasificación de las complicaciones postoperatorias con referencia inicial a las colecistectomías. Plantearon 4 grados: I son alteraciones leves del postoperatorio normal sin efectos duraderos (ejemplo: arritmias, atelectasias, íleo postoperatorio, etc.); grado II son efectos potencialmente fatales, pero sin efectos duraderos (ejemplo: angina, crisis hipertensiva, embolia pulmonar, bacteremia, hemorragia digestiva postoperatoria, drenaje quirúrgicos o radiológicos, etc.); grado III son complicaciones con efectos residuales o persistencia de condiciones eventualmente fatales (ejemplo: infarto miocardio, pancreatitis aguda con diabetes, sección vía biliar, etc.) y grado IV son complicaciones que resultan en fallecimiento del paciente. Posteriormente, en 2004, esta clasificación fue reevaluada críticamente por los autores ${ }^{26}$ y modificada para lograr su aceptación más general, en especial en lo referente a los efectos adversos que son potencialmente fatales o producen complicaciones permanentes. Los autores proponen 5 grados en vez de los 4 originales, pero nos parece bastante más complejo, ya que en total incluye 9 grupos. No estimamos que sea adecuado usarla masivamente entre nosotros por su poca aplicabilidad práctica.

También es muy importante el hecho que en la actualidad han aumentado los pacientes de mayor edad que acceden a cirugías de alta complejidad. En la década del 70 se consideraba a pacientes sobre 65 años como “ancianos” y prácticamente eran excluidos de la cirugía. En la actualidad, como se aprecia en el presente estudio, un tercio de los pacientes tenían más de 70 años. En un estudio reciente publicado por nosotros ${ }^{27}$, se comprobó que en 115 pacientes operados, mayores de 80 años, la cirugía significó hospitalización prolongada, alto costo económico, alta tasa de permanencia en UCI, y alta tasa de reoperaciones, lo que representa un nuevo desafío para los cirujanos. Nuestro actual protocolo de tratamiento de los pacientes sometidos a gastrectomía total incluye la hospitalización 1 día previo a la cirugía para su evaluación anestésica, kinésica y muy en especial, la evaluación nutricional para el manejo pre y postoperatorio $^{28}$. En el pabellón se coloca vía venosa central, analgesia epidural, sonda Foley y compresión mecánica intermitente. La cirugía comprende una gastrectomía total con disección D2 y omentectomía. Siempre se envía un segmento del límite oral a anatomía patológica para evitar infiltración oral del tumor. La anastomosis esófago-yeyunal se realiza 
con Stapler circular 25 y se dejan 2 drenajes. En el postoperatorio se comienza la alimentación enteral a las 24 a 48 hrs y se mantiene por lo menos 5 a 7 días. Entre el $6^{\circ}$ a $7^{\circ}$ día se realiza control radiológico con sulfato de bario para evaluar la anastomosis.

Si no hay conflicto, el paciente se da de alta al $7^{\circ}$ o $9^{\circ}$ día. Planteamos que la adopción de este estricto protocolo permite llegar a estas cifras de mortalidad, al optimizar el tratamiento quirúrgico de pacientes con cáncer gástrico.

\section{Referencias}

1. Rajs D. Estadísticas de mortalidad por tumores malignos. MINSAL Chile.

2. Csendes A. Estado actual del tratamiento del cáncer gástrico. Rol de la Linfadenectomía. Cuad Cir. 1994;8:8291.

3. Csendes A, Burdiles P, Rojas J, Braghetto I, Díaz JC, Maluenda F. A prospective randomized study comparing D2 total gastrectomy versus D2 total gastrectomy plus splenectomy in 187 patients with gastric carcinoma. Surgery 2002;131:401-7.

4. Csendes A, Braghetto I, Burdiles P, Debandi A, Díaz JC, Rappoport J. Estudio prospectivo y randomizado de 2 tipos de anastomosis como método de reconstrucción después de gastrectomía total. Rev Chil Cir. 1994;46:348-51.

5. Csendes A, Korn O, Burdiles P, Braghetto I, Díaz JC, Maluenda F. Comparación de la anastomosis esófagoyeyunal mecánica versus manual para gastrectomía en pacientes con cáncer gástrico. Rev Chil Cir. 1994;46:5962.

6. Csendes A, Díaz JC, Burdiles P, Braghetto I, Maluenda F, Nava O. Classification and treatment of anastomotic leakages after extended total gastrectomy in gastric carcinoma. Hepatogastroenterology 1990;37:174-7.

7. Csendes A, Burdiles P, Braghetto I, Díaz JC, Maluenda F, Korn O., y cols. Resecabilidad y mortalidad operatoria de la gastrectomía subtotal y total en pacientes con cáncer gástrico avanzado entre 1969 y 2004. Rev Med Chile 2006;134:426-32.

8. Otaiza E, Lopetegui G, Csendes A. Operabilidad y Resecabilidad del cáncer gástrico. Rev Med. Valpo. 1969;22:228-30.

9. Csendes A, Díaz H, Santa Cruz V, Flores N. Resultados inmediatos de la cirugía en el cáncer gastroesofágico. Arch Soc Cir Chile 1972;24:142-6.

10. Csendes A, Flores N. Gastrectomía total: Resultados inmediatos y sobrevida. Arch. Soc Cir Chile 1974;26:2448.

11. Csendes A. Gastric cancer in Chile. A cooperative inter hospital study. Jap J Clin Oncol. 1975;5:47-52.

12. Csendes A, Flores N. Cáncer gastrico: Estudio coope- rativo inter hospitalario II parte. Tratamiento, hallazgos anátomo quirúrgicos y evolución postoperatoria. Arch Soc Cir Chile 1975;27:302-8.

13. Csendes A, Caracci M, Pari G, Pavés J, Venturelli A. Aspectos clínicos y terapéuticos del cáncer gástrico 1973-1979. Rev Med Chile 1983;111:262-7.

14. Braghetto I, Lazo M, Rodríguez A, Amat JI, Bardavid A. Cirugía complementaria con quimioterapia en dosis mínimas efectiva como tratamiento del cáncer gástrico. Rev Chil Cir. 1989;41:226-32.

15. Anselmi M, Mendoza, Hormazabal T, Pari G, Oyara R, Fuentes V. Cáncer gástrico avanzado. Tratamiento quirúrgico y evolución postopertoria. Rev Chil Cir. 1989;41:45-9.

16. Álvarez R, Davanzo A, Funke R, Pozo R, Castillo M, Klaasen R, y cols. Gastrectomía total ampliada. Rev Chil Cir. 1995;47:41-6.

17. Venturelli A, Nivea A, Díaz J, Kushel C, Hermosilla P, Avendaño R. Esofagoyeyunoanastomosis con sutura continua en gastrectomía total por cáncer gástrico. Rev Chil Cir. 1996;48:348-51.

18. Burmeister R, García C, Benavides C, Apablaza S. Fístulas de la anastomosis esófago yeyunales. Experiencia en 10 años. Rev Chil Cir. 1997;49:402-5.

19. Cenitagoya G, Berghi C, Keniger J, Casanueva D. Cáncer gástrico. Sobrevida real a 5 años. Rev Chil Cir. 1997;49:629-32.

20. Waugh E, Hamilton J, Caracci M, De La Fuente H, Díaz A. Cirugía docente en cáncer gástrico: 100 casos consecutivos. Rev Chil Cir. 1999;51:265-70.

21. Hernández F, Matus C, Cerda R, Álvarez S, Leiva L, Cortés L. Gastrectomía total en cáncer gástrico. Resultados inmediatos. Rev Chil Cir. 1999;51:259-64.

22. Álvarez R, Seguel E, Molina H, Betancur G, Gallardo AM, Gallardo O. Tratamiento quiúrgico del cáncer del tercio proximal del estómago y cardias. Rev Chil Cir. 1999;51:405-12.

23. Álvarez R, Funke R, Molina H, Cancino A, Torres D, Durán R. Gastrectomía total en pacientes mayores de 65 años. Rev Chil Cir. 2004;56:443-8.

24. Valenzuela E, Martínez R, Sharp A, Nogueira C, Roblero HL. Interposición de asa tipo Herley-Longuine en gastrectomía total. Rev Chil Cir. 2004;56:449-51.

25. Clavien PA, Samabria JR, Strasberg SM. Proposed classification of complications of Surgery with examples of utility in cholecystectomy. Surgery 1992;111:518-26.

26. Dindo D, Demartines N, Clavien PA. Classification of surgical complications. Ann Surg. 2004;240:205-13.

27. Csendes A, Lembach H, Molina JC, Inostroza G, Kobrich S. Cirugía en mayores de 80 años. Evolución clínica y costos asociados. Rev Chil Cir. 2010;62:564-9.

28. Papapietro K, Díaz E, Csendes A, Díaz JC, Burdiles P, Maluenda F. Nutrición enteral precoz en pacientes con gastrectomía total por cáncer gástrico. Rev Med Chile 2002;130:1125-30. 